INPLASY

PROTOCOL

To cite: Jiang et al. Can Chinese herbal medicine reduce amputation events of diabetic foot ulcers? A protocol for systematic review and meta-analysis. Inplasy protocol 202130012. doi: 10.37766/inplasy2021.3.0012

Received: 06 March 2021

Published: 06 March 2021

Corresponding author:

Xiao-po Tang

tangxiaopo@163.com

Author Affiliation:

Guang'anmen Hospital, China

Academy of Chinese Medical

Science

Support: National Natural

Science.

Review Stage at time of this submission: Preliminary searches.

Conflicts of interest:

None declared.

\section{Can Chinese herbal medicine reduce amputation events of diabetic foot ulcers? A protocol for systematic review and meta-analysis}

Jiang, W1; Xia, C2; Jiang, Q3; Xu, H4; Cheng, Z5; Li, D6; Peng, Q7; Tang, $X^{8}$.

Review question / Objective: We will search, with no time restrictions, electronic databases, contact with authors, trial registers, or grey literature.

Condition being studied: Diabetic foot ulcers (DFU) are one of the serious complications of diabetes; these ulcers develop in about $15-25 \%$ of patients with diabetes. In addition, $28 \%-89 \%$ of amputations performed for nontraumatic lesions are associated with diabetes. DFU cause not only pain problems and disability, but also heavy emotional and economic burdens. The global burden of DFU is expected to be raised, because of the increasing incidence of diabetes. Therefore, it is necessary and urgent to find some cost-effective treatments for DFU. Multidisciplinary treatment of DFU has been suggested, because DFU is such a complicated condition. Chinese herbal medicine (CHM) has been used as an adjunctive method for managing DFU. Some clinical trials evaluated the role of traditional CHM for treating DFU. Their results showed that CHM may improve the conditions of patients with DFU. However, the answer to the question that if $\mathrm{CHM}$ is effective as an adjunctive therapy for reducing amputation of DFU is not confirmed.

INPLASY registration number: This protocol was registered with the International Platform of Registered Systematic Review and Meta-Analysis Protocols (INPLASY) on 06 March 2021 and was last updated on 06 March 2021 (registration number INPLASY202130012).

\section{INTRODUCTION}

Review question / Objective: We will search, with no time restrictions, electronic databases, contact with authors, trial registers, or grey literature.
Condition being studied: Diabetic foot ulcers (DFU) are one of the serious complications of diabetes; these ulcers develop in about $15-25 \%$ of patients with diabetes. In addition, $28 \%-89 \%$ of amputations performed for nontraumatic 
lesions are associated with diabetes. DFU cause not only pain problems and disability, but also heavy emotional and economic burdens. The global burden of DFU is expected to be raised, because of the increasing incidence of diabetes. Therefore, it is necessary and urgent to find some cost-effective treatments for DFU. Multidisciplinary treatment of DFU has been suggested, because DFU is such a complicated condition. Chinese herbal medicine (CHM) has been used as an adjunctive method for managing DFU. Some clinical trials evaluated the role of traditional CHM for treating DFU. Their results showed that $\mathrm{CHM}$ may improve the conditions of patients with DFU. However, the answer to the question that if CHM is effective as an adjunctive therapy for reducing amputation of DFU is not confirmed.

\section{METHODS}

Participant or population: Adults with diabetic foot ulcers (as diagnosed by a clinician, or using any recognized diagnostic criteria) will be included. Adults with other types of foot ulcers (ulcers caused by venous disease, vasculitis, or any conditions other than diabetes) will be excluded.

Intervention: Chinese herbal medicine (CHM) combined with standard wound therapy (SWT) is the intervention.

Comparator: The standard wound therapy (SWT) are defined as standard antidiabetic treatment with or without use of antibiotics and debridement.

Study designs to be included: Only randomized controlled trials (RCTs) will be included. Nonrandomized controlled trials, observational studies, and cohort studies were ruled out.

Eligibility criteria: Trials will be included that evaluated use of CHM combined with standard therapy for DFU. The mode of delivery (for example oral, topical use or both), dosage, and regimen of herbs will be not restricted. We included trials if the treatment was given for a minimum of four weeks. The standard wound therapy (SWT) are defined as standard antidiabetic treatment with or without use of antibiotics and debridement. Trials using CHM combined with treatment options other than standard therapy will be excluded. These treatment options referre to pressure-relieving interventions, decompressive surgery, etc.

Information sources: We will search, with no time restrictions, electronic databases, contact with authors, trial registers, or grey literature.

Main outcome(s): The primary outcome is amputation event as a long-term outcome. The effect measure is to treat the number of amputation events.

Additional outcome(s): Secondary outcomes are complete healing of the wound and healing time.

Quality assessment / Risk of bias analysis: Two reviewers independently assessed the methodological quality of the included studies using the 5-point Jadad scale.

Strategy of data synthesis: The data were analyzed with Review Manager 5.3. Comparisons of amputation events were made between standard therapy combined with CHM and standard therapy used alone.

Subgroup analysis: Comparisons of complete Healing events were made between standard therapy combined with CHM and standard therapy used alone.

Sensitivity analysis: The purpose of sensitivity analysis is to eliminate lowquality studies to reduce heterogeneity. By excluding a certain study to observe whether there is a significant change in heterogeneity, the reliability, and stability of the results can be evaluated.

Language: English and Chinese.

Country(ies) involved: China. 
Keywords: diabetic foot; Chinese medicine; amputation; systematic review.

Contributions of each author:

Author 1 - Wen Jiang - The author drafted the manuscript.

Email: drjiangwen@163.com

Author 2 - Cong-min Xia - The author provided statistical expertise.

Email: xiacongmin@126.com

Author 3 - Quan Jiang - The author provided statistical expertise.

Email: doctorjq@126.com

Author 4 - Hao-dong Xu - The author contributed to the development of the selection criteria, and the risk of bias assessment strategy.

Email: xhd2015057001@163.com

Author 5 - Zeng-yu Cheng - The author contributed to the development of the selection criteria, and the risk of bias assessment strategy.

Email: wsczyvip@163.com

Author 6 - Da Li - The author contributed to the development of the selection criteria, and the risk of bias assessment strategy.

Email: lida1768@163.com

Author 7 - Qiu-wei Peng - The author contributed to the development of the selection criteria, and the risk of bias assessment strategy.

Email: M18260095353@163.com

Author 8 - Xiao-po Tang - The author read, provided feedback and approved the final manuscript.

Email: tangxiaopo@163.com 